\title{
On Semantic Equivalence in English-Chinese Translation
}

\author{
Linyan $\mathrm{Fu}$
}

\author{
Jiangxi Science \& Technology Normal University, Jiangxi Nanchang 330038
}

\author{
Keywords: English-Chinese translation; Semantic equivalence; Pragmatics preset; Associated \\ factors
}

\begin{abstract}
Translation is an advanced subject and semantic equivalence in translation has always been the focus of the debate of scholars. How to achieve semantic equivalence becomes a hot spot in the focus. Due to cultural differences and expression habits, semantic equivalence is almost completely impossible. But it is possible to achieve maximum degree of semantic equivalence, if considering various factors and taking the appropriate strategy as much as possible in translation. This paper tries to analyze the semantic equivalence problem in English-Chinese translation from five aspects, such as theory connotation, influencing factors, realizability, relativity and countermeasures of semantic equivalence, and proves equivalence theory is of extensive significance as the standard and principle in translation.
\end{abstract}

\section{Introduction}

English-Chinese translation is closely associated with context and it is difficult to have a comprehensive and accurate understanding of the exact meaning of a text from the sentence literally. On the one hand, the meaning of the text is implicit, which is hidden in the discourse context or in the minds of speakers or receivers, which is the cultural background knowledge and ideas of both sides. In English-Chinese translation, the former will not constitute a barrier on translation; but the latter case, due to the differences between source language and target language, the meanings of the source text the author wants to convey is not commonly known by the readers of the target language, which causes difficulties in translation. Under this situation, racking brains, translators make every effort to find the best translation method painstakingly which can convey source meanings and try to minimize the losses. However, the translation often attends to one thing and loses another and leave regrets.

To sum up, the textual meanings have close relationship with speakers, receivers and the context. In other words, to find the accurate understanding of the meanings of a text, the belief, attitude and intention of the speaker must be considered.

Semantic equivalence is an advanced translation equivalence. Before understanding semantic equivalence problem, translators must understand what semantic is and what semantic equivalence is. To understand the research status and significance of semantic equivalence has important guiding role in understanding the theory. While all this is closely connected with pragmatics preset. For this reason, this paper does some analysis of pragmatics preset in English-Chinese translation.

\section{Theory Connotation of Semantic Equivalence}

Semantic equivalence is an advanced translation equivalence. Before understanding semantic equivalence problem, translators must understand what semantic is and what semantic equivalence is. To understand the research status and significance of semantic equivalence has important guiding role in understanding the theory.

Semantic. Generally speaking, the semantic is the meanings of a language. In translation, it refers to the meaning of a word or a sentence. Semantic is from words equivalence until equivalence terminates and it is throughout the whole process of translation. Then, what is "meaning"? American translation theorist Nida summarized "meaning" in translation into "semantic" and "style" when he gave definition of translation, he said, "Translating consists in reproducing in the receptor language the closest natural equivalent of the source- language message, 
first in terms of meaning and secondly in terms of style." The so-called translation is to reproduce the information of the source language in translation by the most natural equivalent language from semantic to style. Here, Nida uses the word "information" which is first reflected in "semantic". It is clear that the meanings of the target language should be as far as possible consistent with that of the source language. Semantic equivalence is to strive to realize the most basic equivalence in the process of translation.

Semantic Equivalence. Semantic equivalence is the deep equivalence in translation based on surface equivalence of translation, such as equivalences of format, rhythm, part of speech, grammar, etc. Semantic equivalence emphasizes the translation and the source text should achieve equivalent results in the expression. The hidden deep meanings in source text should be reflected in translation text. For example, the feelings which the author convey and the touches which the feelings bring to readers can make the readers of the two languages achieve the same position when appreciating the work of different languages. For example, a piece of music can bring people of different countries the same feelings without borders. Semantic equivalence is to make languages produce this kind of feelings without borders.

\section{Associated Factors of Semantic Equivalence}

To realize semantic equivalence, many factors need to be taken into consideration. The comprehensive understanding of the cultural difference between the two languages can make translators grasp the surface and penetrate the skin texture of the paper, as well as deeply understand its cultural connotation and show the vivid words to the foreign readers. Specifically, the associated factors of semantic equivalence mainly include the following several aspects.

Historical and Cultural Differences. Every country has its own cultural background and historical and cultural heritage and development have big differences. It is the historical and cultural differences make people have big differences in the understanding of things. The typical example is that the meanings of "dog" are different in English and Chinese. In Chinese, we will use "mad dog bites people", "dog bites Lv Dongbin which means someone is not grateful", "be a bully under the protection of a powerful person" and other phrases. In Chinese culture, "dog" is often derogatory. On the contrary, in English language and culture, "dog" is a friend of human, so, its usage is different with us. They often say: "The old dog will not earn new things" which means old dog cannot learn new things apparently and actually means old man is very difficult to adapt to new things.

Customs and Habits Differences. In English-speaking countries, people's customs and habits are different from us. From the point of geographical position, Britain is an island country which has developed fishing and sailing industry. So in its language, there are many vocabulary which is related to sea and sailing. For example, "a ship in the sea" means meeting someone by chance and "to teach a fish to swim" means displaying one's slight skills before an expert. The direct translation to "a ship in the sea" and "to teach a fish to swim" is not consistent with the context. The difference of customs and habits makes it hard for us to understand each other's language, which is an important factor of semantic equivalence.

Religious Belief Differences. Religious belief is an important spiritual pillar of every country, which profoundly affects people. In English culture, the number of Christians is large. So "god" is commonly used in English. Under the influence of Confucian culture, Chinese often use "regality dominated by the nature", "son of the heaven" and "destiny of the heaven". Chinese often use "Man proposes, the heaven disposes", while English-speaking countries use "Oh, my god." and "For God's sake." If translators are lack of understanding of the religion differences of the two countries, it is hard to understand the profound connotation of the two languages.

\section{The Connotation and its Role in English-Chinese Translation of Pragmatics Preset}

Definition of Pragmatic Preset. The concept of "preset" was first suggested by German philosopher Frege in 1892. And preset is divided into narrow one and broad one, and the preset of 
pragmatics is generalized as broad one with characteristics of strong context and dynamic. Pragmatics preset is also called pragmatic premise, which is actually a kind of pragmatic inference and generally refers to the known information of both communication sides, or the information which can be inferred at least according to the context. Thus, to determine the pragmatics preset of a text in a specific context, it is necessary to determine the proposition of the text in specific context. To determine the specific meaning of a text in specific context, specific methods shall be taken according to specific context. For example, in oral English, specific meanings can be judged according to the different stresses in a sentence. So, what functions does pragmatics preset have in English-Chinese translation?

The Role of Pragmatic Preset in English-Chinese Translation. English-Chinese translation is the communication between two kinds of culture of Chinese and English. Therefore, English-Chinese translation must take English and Chinese culture as the premise. The functions of pragmatics preset in English-Chinese translation are reflected in the three layers, such as integrity, effectiveness and purpose. Specifically, integrity is that the source language culture exists in the following three space, such as the cognitive context of the target readers, inside the target language and outside the target language as an organic whole of the translation. Therefore, translators should not only adopt flexible translation strategies according to the target language readers' cultural experience, but also consider the source language culture. In general, the integrity function of pragmatic preset is translation must effectively understand the meanings of source language and make sure there is no missing information of the culture in source language. The effectiveness is target language readers can understand and grasp the meanings of the source language effectively, as well as effectively carry out culture communication. And purpose refers to translators shall transfer cultural information and the creation of the original intention of the source language as far as possible to the target language readers. It can be seen that in English-Chinese translation, the cultural factors contained in the source work shall be given full consideration, especially the preset of pragmatics. Only then can English-Chinese translation completely transfer cultural information between English and Chinese.

\section{Conclusion}

Throughout the full paper, we know that pragmatics preset is close to context, beliefs, attitudes, intentions, etc. of the people engaged in the communication. Obviously, pragmatics preset is indispensable in English-Chinese translation. The success of English-Chinese translation largely depends on the understanding of pragmatics preset. Systemic Functional School considers language context, meaning and lexical grammar are interdependent and influence each other. When understanding the source language text, the translator does not only have to understand the meanings of vocabulary and grammar, but also to understand the context to grasp the meaning of pragmatics preset and determine the choice of words through context. Therefore, after proper reasoning and combining the analysis of the context through pragmatics preset, the translator can correctly understand the connotation of the source language text, so as to avoid mistranslation and translation errors in the process of English-Chinese translation.

\section{References}

[1] Nelson T O, Dunlosky J. Norms of paired-associate recall during multitrial learning of Swahili-English translation equivalents.[J]. Memory, 1994, 2(3):325-335.

[2] Frank B. On the nutritional dependence of certain trees on root symbiosis with belowground fungi (an English translation of A.B. Frank' s classic paper of 1885)[J]. Mycorrhiza, 2005, 15(4):267.

[3] Wang N, Chinglish in Chinese-English Translation[J]. Chinese Translators Journal, 2000. 
[4] Pomeroy S. Oeconomicus: A Social and Historical Commentary, with a New English Translation[J]. Clarendon Paperbacks, 1996.

[5] by John Von Neumann. Zur Theories der Gesellschaftsspiele," Mathematische Annalen, 100, pp. 295 - 320. English translation by[C]// Contributions to the Theory of Games IV. 2015.

[6] Falola U U T. A Comparison of Jacob Egharevba's Ekhere Vb Itan Edo and the Four Editions of Its English Translation, A Short History Of Benin[J]. History in Africa, 1998, 25:361-386.

[7] Hertz B H. On the contact of elastic solids. Zeitschrift fur Reine und Angewandte Mathematik 92, 156--171 (1882). English translation[C]// Miscellaneous Papers. 2010.

[8] Levistrauss B C. English translation 1969, The Elementary Structures of Kinship[J]. 2010.

[9] RENJing-sheng. A Further Discussion on English Translation of Chinese Dishes and Foods[J]. Hn Ranlaor Jornal, 2001.

[10] GómezPalacio, Manuel, VillazónS, et al. English Translation: El Efecto De Diversos Rangos De Aporte Calorico Y Nitrogenado the Effect of Different Levels of Caloric and Nitrogenous Contribution[J]. Critical Care Medicine, 1981, 9(3).

[11] Y.A Tao, A tentative study on a new model of compiling Chinese-English translation textbooks for translation majors[J]. Foreign Language World, 2008.

[12] Nietzsche F W. The Complete Works of Friedrich Nietzsche: The First Complete and Authorised English Translation[J]. Friedrich Nietzsche, 1911, 41(1):54-56. 